\title{
Reliability and Validity of the Turkish Language Version of the International Consultation on Incontinence Questionnaire - Male Lower Urinary Tract Symptoms
}

\author{
Oğuz Mertoğlu', Oktay Üçer², Yasin Ceylan ${ }^{3}$, Ozan Bozkurt', Bülent Günlüsoy ${ }^{3}$, Ali Can Albaz², Ömer Demir ${ }^{4}$; Aegean Study \\ Group of Society of Urological Surgery
}

${ }^{1}$ Department of Urology, Izmir Tepecik Training and Research Hospital, İzmir, Turkey

${ }^{2}$ Department of Urology, Celal Bayar University, Faculty of Medicine, Manisa, Turkey

${ }^{3}$ Department of Urology, Izmir Bozyaka Training and Research Hospital, İzmir, Turkey

${ }^{4}$ Department of Urology, Dokuz Eylül University, Faculty of Medicine, İzmir, Turkey

\begin{abstract}
Purpose: Patients receiving treatment for benign prostate hyperplasia may have persistent storage symptoms. There has been increasing debate on the precision and accuracy of the International Prostate Symptom Score questionnaires over other questionnaires in evaluating all the complaints of lower urinary tract symptoms (LUTS). The aim of this study was to perform the validity and reliability analysis of the Turkish version of the Internatinal Consultation on Incontinence Questionnaire-Male LUTS (ICIQ-MLUTS).

Methods: Linguistic validation was studied (i.e., translation and back translation). Intelligibility was completed between October 2013 and November 2013. Data was collected between November 2013 and March 2014. The stability and reliability of the form were measured with the Cronbach test.

Results: In total, 117 male patients were included in the study. At the first visit, the mean age of the patients was 59 years (range, 18-84 years). For reliability, the Cronbach alpha value was 0.798 , demonstrating the internal consistency of the form $(r>0.7)$. The internal consistency of each question was examined separately and found to be over 0.7. For the evaluation of test-retest reliability, the test was administered to 116 patients for a second time with an interval of 2-4 weeks. The first and second test scores for each question were found to be correlated $(\mathrm{r}=0.741)$.

Conclusions: ICIQ-MLUTS is a new questionnaire, which can be used for evaluating male LUTS in Turkey. We believe that the Turkish version of the ICIQ-MLUTS is an important breakthrough in our country.
\end{abstract}

Keywords: Questionnaire; Lower Urinary Tract Symptoms; Male; Validation

- Research Ethics: This study was approved by Institutional Review Board of Celal Bayar University (approval number: 20478486-178).

- Conflict of Interest: No potential conflict of interest relevant to this article was reported.

\section{INTRODUCTION}

Lower urinary tract symptoms (LUTS) refers to a cluster of symptoms experienced by patients with complaints of the lower

Corresponding author: Oktay Üçer (iD http://orcid.org/0000-0001-7912-0408 Department of Urology, Celal Bayar University, Faculty of Medicine, Manisa, Turkey

E-mail: uceroktay@yahoo.com / Tel: +905052114618 / Fax: +902362338040

Submitted: October 8, 2015 / Accepted after revision: November 30, 2015 urinary tract, including overactive bladder, urinary incontinence, interstitial cystitis, and benign prostatic hyperplasia (BPH) [1]. The International Prostate Symptom Score (IPSS), which is a widely used questionnaire to assess LUTS in male 
patients, evaluates incomplete emptying, frequency, intermittency, urgency, weak stream, hesitancy, and nocturia [2,3]. Additionally, it aids in determining other associated symptoms in the quality of life (QoL) section. However, there has been increasing debate on the precision and accuracy of the IPSS [3-5]. It does not comprehensively assess all LUTS, particularly storage symptoms (e.g., incontinence) as described by patients [5]. The Danish Prostatic Symptom Score (DAN-PSS) is another known questionnaire that evaluates storage symptoms, including incontinence, in a more detailed manner than IPSS. The DAN-PSS is recommended as level B by the International Consultation on Male LUTS [6,7]; however, it remained popular only in Scandinavian countries.

The International Consultation on Incontinence Questionnaire-Male LUTS (ICIQ-MLUTS) has been created from the International Continence Society (ICS) Male Questionnaire, which is a product of the ICS-BPH Study [8]. Currently, the ICIQ-MLUTS is a highly recommended, patient-completed questionnaire used for evaluating male LUTS. Six storage symptoms, as well as voiding symptoms, can be assessed using ICIQ-MLTUS. It also provides individual bother scores associated with the present symptoms [7]. The aim of this study was to analyze the validity and reliability of the Turkish version of ICIQ-MLUTS.

\section{MATERIALS AND METHODS}

\section{Original ICIQ-MLUTS Questionnaire}

The ICIQ-MLUTS is a recently developed questionnaire that interprets male LUTS and their impact on patients' QoL. Its main advantage over previously used questionnaires is its capability of evaluating the voiding and storage symptoms, as well as continence status and their impact on patients' QoL one by one. The unique property of this tool can help in discussing treatment alternatives in a more detailed manner with the patient.

\section{Methodology}

The senior author (O.D.) contacted the owner of this questionnaire, Nikki Cotterill, on behalf of the ICIQ group for linguistic validation. After acquiring approval from the Institutional Review Board of Celal Bayar University (approval number: 20478486-178), the English version of the questionnaire was first translated into Turkish by an independent medical translator. The authors reviewed the translated questionnaire for medical coherence. Then, another independent medical translator who had never met the first translator retranslated the Turkish version into English. This version was then checked by Nikki Cotterill, and the final Turkish version was developed according to the suggested recommendations.

\section{Control of Intelligibility}

Nikki Cotterill provided an interview schedule to check the intelligibility of all the items in the questionnaire with questions, such as "How easy or difficult did you find the questionnaire to complete?"; "Were the items clear? If not, which ones and why?"; "What do you feel about the length of the questionnaire?"; and "Are there any further comments you would like to make or items you would like to ask about?"; and the scheduled interview was performed for intelligibility control. The control of intelligibility interviews were performed with 13 patients with LUTS, as suggested by the questionnaire owner. Initially, they were asked to complete the questionnaire on their own. Then, each item was evaluated via face-to-face interview with the patients for possible misunderstandings, as well as for fluency and smoothness. Using the patients' feedback, a final Turkish version was produced by the authors.

\section{Participant Recruitment and Data Collection}

Patients aged 18-70 years suffering from LUTS were recruited from 4 outpatient clinics between November 2013 and March 2014. A demographic and clinical information form and the Turkish version of ICIQ-MLUTS were administered at the first visit. At the second visit, the questionnaire's stability was assessed with a test-retest reliability analysis, where the same set of respondents filled out the questionnaire for a second time, 2-4 weeks after the initial assessment. We chose this period with the expectation that the symptoms would remain stable and that the respondents would not likely remember their first responses.

\section{Statistical Analysis}

Statistical analyses were performed using the SPSS ver. 15.0 (SPSS Inc., Chicago, IL, USA). Internal consistency was determined by calculating Cronbach alpha coefficient. Test-retest reliability was analyzed with intraclass correlation coefficient (ICC). ICC values $>0.7$ were accepted as satisfactory [9]. The minimum acceptable value for Cronbach alpha coefficient was 0.7. A value of $\mathrm{P}<0.05$ was accepted as statistically significant. 


\section{RESULTS}

In total, 117 male patients were included in the study. The mean age of the patients was $58.9 \pm 10.5$ years. Regarding the level of education, 30 participants (25.6\%) held a university degree, 52 (44.4\%) graduated from high school, and 35 others (29.9\%) completed primary education. All participants agreed that the questionnaire was clear, appropriate, and easy to understand when interviewed at the end of their first visit.

Domain scores of ICIQ-MLUTS questionnaire of the patients are shown in Table 1.

For reliability, Cronbach alpha value, which is indicative of internal consistency, was found as 0.798 , which indicated that the questionnaire was internally consistent. The internal consistency of each question was examined separately; all questions had values over 0.7 (Table 2).

For evaluating test-retest reliability, the test was administered

Table 1. Domain scores of ICIQ-MLUTS questionnaire

\begin{tabular}{llc}
\hline ICIQ-MLUTS questionnaire & No. & Mean \pm SD \\
\hline Voiding score & 117 & $9.3 \pm 4.3$ \\
Incontinence score & 117 & $4.5 \pm 3.5$ \\
Frequency & 117 & $1.2 \pm 1.1$ \\
Nocturia & 117 & $2.3 \pm 1.1$ \\
\hline
\end{tabular}

ICIQ-MLUTS, Internatinal Consultation on Incontinence Questionnaire-Male Lower Urinary Tract Symptoms; SD, standard deviation.

Table 2. Correlations between each question and total score and Cronbach alpha value of each question if item deleted

\begin{tabular}{lcc}
\hline Symptom & $\begin{array}{c}\text { Item-total score } \\
\text { correlation }\end{array}$ & $\begin{array}{c}\text { Cronbach alpha } \\
\text { if item deleted }\end{array}$ \\
\hline Hesitancy & 0.637 & 0.775 \\
Starting strain & 0.602 & 0.780 \\
Decreased stream & 0.631 & 0.776 \\
Intermittency & 0.656 & 0.773 \\
Incomplete emptying & 0.502 & 0.791 \\
Urgency & 0.591 & 0.781 \\
Urge incontinence & 0.533 & 0.785 \\
Stress incontinence & 0.424 & 0.792 \\
Unpredictable incontinence & 0.390 & 0.794 \\
Nocturnal incontinence & 0.262 & 0.804 \\
Postvoid dribbling & 0.598 & 0.780 \\
Frequency & 0.571 & 0.782 \\
Nocturia & 0.539 & 0.786 \\
\hline
\end{tabular}

to 116 patients for the second time, with an interval of 2-4 weeks. The mean domain scores of ICIQ-MLUTS for the first and second visits are given in Table 3. The total agreement percentage was $62.4 \%$, and the mean correlation value, 0.741 (Fig. 1).

When the bother scales for each symptom were evaluated, we found that nocturia and incomplete emptying were the most bothering symptoms. Unpredictable incontinence and nocturnal incontinence were the least bothering symptoms.

\section{DISCUSSION}

The management strategy for men with LUTS has recently gained a different dimension. During the 90 's, enlarged prostate, urinary symptoms, and bladder outlet obstruction were the main subjects of discussion in the management of men with LUTS. Nowadays, bladder dysfunction, polyuria, and outflow tract pathology are mainly involved in the evaluation of male LUTS [7].

Table 3. The mean domain scores of ICIQ-MLUTS for first and second visits

\begin{tabular}{lcc}
\hline Variable & First visit & Second visit \\
\hline Voiding score & $9.3 \pm 4.3$ & $9.3 \pm 4.3$ \\
Incontinence score & $4.5 \pm 3.5$ & $5.1 \pm 3.7$ \\
Frequency & $1.2 \pm 1.1$ & $1.3 \pm 1.0$ \\
Nocturia & $2.3 \pm 1.1$ & $2.3 \pm 1.1$ \\
\hline
\end{tabular}

Values are presented as mean \pm standard deviation. ICIQ-MLUTS, Internatinal Consultation on Incontinence Questionnaire - Male Lower Urinary Tract Symptoms.

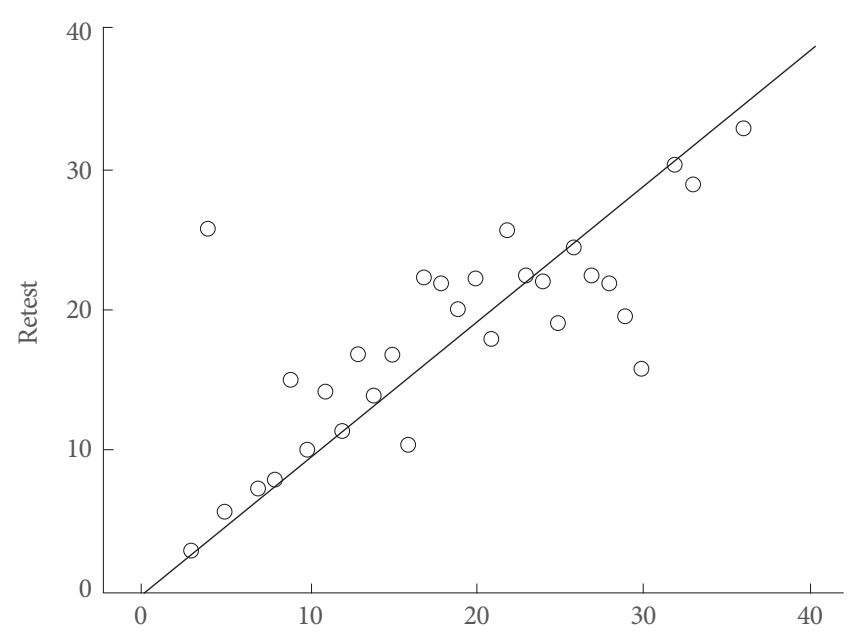

Fig. 1. Correlation graph of the test-retest results. 
In the EpiLUTS study, it has been emphasized that a more expansive view of LUTS looks beyond an organ-specific focus and the interaction of the whole urinary system in both men and women, and it was suggested that all these symptoms should be evaluated completely [10]. This indicates the need for comprehensive questionnaires.

LUTS in men can be classified as storage symptoms, voiding symptoms, and postvoiding symptoms. Storage symptoms are more prominent in both men and women presenting with LUTS [10]. In our country, LUTS in men is commonly evaluated using the IPSS questionnaire. Although this form is recommended as grade A, it is not as efficient in evaluating every information relating to LUTS, especially for storage symptoms like incontinence and overactive bladder symptoms. Despite using the word "prostate" in its title, the IPSS should not be taken as a condition-specific form, particularly for prostate [7].

A recent community study reported that the most distressing LUTS in men were urgency and nocturia. Postmicturition dribbling and urge urinary incontinence were other bothersome problems. The efficiency of IPSS has been a subject of debate since it has low to moderate correlation with frequency-volume charts and has only one QoL question with a likert scale [5]. This is another possible reason for exploring the need of a more comprehensive questionnaire.

In 1996, the ICS-male questionnaire was produced according to the ICS-BPH study. The long form has been reduced to a simpler type. ICS male short form contains questions on symptoms that cause the highest levels of problem or bothersomeness, which is incontinence like the DAN-PSS. However, unlike the IPSS it is scored simply by adding all the individual scores. This form was then developed into the ICIQ-MLUTS form. Although highly recommended, the ICIQ-MLUTS is not as popular as the IPSS. The module of ICIQ-MLUTS consists of 13 questions, evaluating 6 storage symptoms, 5 emptying symptoms, and additional frequency and nocturia questions. Each question has a 4-point severity scale (0-4). Each question is also supplemented with a 10-point QoL visual analog scale (0-10) question, which adds an extra intensity effect to the questionnaire $[7,8,11,12]$.

We aimed to provide validation for the Turkish language version of the ICIQ-MLUTS and to use this form in the management of male LUTS in Urology clinics in Turkey. During the validation steps of the ICIQ-MLUTS, in the translation-back translation part, the questions were observed to be clear and simple in Turkish. A few changes were applied to the question- naire after the intelligibility interview step. When we looked at the age and education groups, it was observed that the patients could adapt easily to the questionnaire. We observed significant results of internal consistency for the validity step. The Cronbach alpha value of the complete questionnaire was 0.798. In the separate analysis of each question, Cronbach alpha values were over 0.7. During the test-retest period, significant correlation and agreement values were found. These results demonstrate the strong internal consistency of the ICIQ-MLUTS in Turkish.

The validated Turkish version of ICIQ-MLUTS will help in evaluating male LUTS in Turkey. We believe that the Turkish version of ICIQ-MLUTS is a new breakthrough in our country in the new era of the management of male LUTS.

\section{ACKNOWLEDGEMENTS}

The Study Group of The Society of Urological Surgery would like to thank to Dr. Nikki Cotterill and the ICIQ group for their kind cooperation during the study period.

\section{REFERENCES}

1. Abrams P, Cardozo L, Fall M, Griffiths D, Rosier P, Ulmsten U, et al. The standardisation of terminology in lower urinary tract function: report from the standardisation sub-committee of the International Continence Society. Urology 2003;61:37-49.

2. Barry MJ, Fowler FJ Jr, O'Leary MP, Bruskewitz RC, Holtgrewe HL, Mebust WK, et al. The American Urological Association symptom index for benign prostatic hyperplasia. The Measurement Committee of the American Urological Association. J Urol 1992;148:154957.

3. Cockett AT, Khoury S, Aso Y. The 2nd International Consultation on Benign Prostatic Hyperplasia (BPH); 1993 June 27-30; Paris, France. Jersey: Scientific Communication International, 1993

4. Liao CH, Chung SD, Kuo HC. Diagnostic value of International Prostate Symptom Score voiding-to-storage subscore ratio in male lower urinary tract symptoms. Int J Clin Pract 2011;65:552-8.

5. Yap TL, Cromwell DA, Brown C, van der Meulen J, Emberton M. The relationship between objective frequency-volume chart data and the I-PSS in men with lower urinary tract symptoms. Eur Urol 2007;52:811-8.

6. Meyhoff HH, Hald T, Nordling J, Andersen JT, Bilde T, Walter S. A new patient weighted symptom score system (DAN-PSS-1). Clinical assessment of indications and outcomes of transurethral prosta- 
tectomy for uncomplicated benign prostatic hyperplasia. Scand J Urol Nephrol 1993;27:493-9.

7. Bosch R, Abrams P, Cotteril N, Gotoh M, Nitti V, Novara G, et al. Lower urinary tract symptoms in men: etiology, patient assessment and predicting outcome from therapy. In: Chapple C, Abrams P, editors. Male lower urinary tract symptoms (LUTS). An International Consultation on Male LUTS; 2012 Sep 30-Oct 4; Fukuoka, Japan. Montreal: Societe Internationale Urologie (SIU); 2013. p. 37-133.

8. Donovan JL, Abrams P, Peters TJ, Kay HE, Reynard J, Chapple C, et al. The ICS-'BPH' Study: the psychometric validity and reliability of the ICSmale questionnaire. Br J Urol 1996;77:554-62.

9. DeVon HA, Block ME, Moyle-Wright P, Ernst DM, Hayden SJ, La- zzara DJ, et al. A psychometric toolbox for testing validity and reliability. J Nurs Scholarsh 2007;39:155-64.

10. Kaplan SA, Roehrborn CG, Chapple CR, Rosen RC, Irwin DE, Kopp Z, et al. Implications of recent epidemiology studies for the clinical management of lower urinary tract symptoms. BJU Int 2009;103 Suppl 3:48-57.

11. Donovan JL, Brookes ST, de la Rosette JJ, Peters TJ, Porru D, Kondo A, et al. The responsiveness of the ICSmale questionnaire to outcome: evidence from the ICS-'BPH' study. BJU Int 1999;83:2438.

12. Donovan JL, Peters TJ, Abrams P, Brookes ST, de aa Rosette JJ, Schafer W. Scoring the short form ICSmaleSF questionnaire. International Continence Society. J Urol 2000;164:1948-55. 